\title{
Zur Körperzusammensetzung von neugeborenen Ferkeln der Deutschen Landrasse und des Deutschen Sattelschweines (Kurzmitteilung)
}

\begin{abstract}
Summary
Title of the paper: Body composition in newborn piglets of the breeds German Landrace and German Saddleback (short communication)

Newbom piglets from 15 litters of German Landrace sows (DL) and 19 litters of German Saddleback sows (DS) were used to determine the body weight, body composition, and characteristics of lipid metabolism in the blood. At the date of birth no differences in body weight and body composition of the piglets were found between the two breeds. The piglets of the DS-sows showed in comparison to the piglets of the DL-sows a significant higher content in high density lipoproteins (HDL)-cholesterol in the blood plasma.
\end{abstract}

Key Words: newborn piglets, German Landrace, German Saddleback, body weight, body composition, cholesterol

\section{Zusammenfassung}

An neugeborenen Ferkeln aus Würen von I5 Deutsche Landrasse (DL)-Sauen und 19 Deutsche Sattelschwein (DS)-Sauen unterschiedlicher Abstammung wurden Untersuchungen zum Körpergewicht, zur Körperzusammensetzung und zu speziellen Merkmalen des Fettstoffwechsels im Blut durchgefuhrt. Zwischen den Tieren der beiden Rassen konnten zum Zeitpunkt der Geburt weder Unterschiede im Korpergewicht noch in der Korperzusammensetzung ermittelt werden. Die Geburtsferkel der Rasse DS wiesen im Vergleich zu den Ferkeln der Rasse DL einen signifikant hðheren Gehalt an high density lipoproteins (HDL)-cholesterol im Blutplasma auf.

Schlüsselwörter: Schwein, Deutsche Landrasse, Deutsches Sattelschwein, Körpergewicht, Körperzusammensetzung, Cholesterin

\section{Einleitung}

In einer früheren Arbeit (KUHN u.a., 1997) wurde nachgewiesen, dass schlachtreife Tiere der Rasse Deutsches Sattelschwein (DS) gegenüber gleichgewichtigen Tieren der Deutschen Landrasse (DL) bei langsamerer Lebendgewichtsentwicklung einen signifikant höheren Verfettungsgrad aufweisen. Das unterschiedliche Leistungspotential der Tiere beider Rassen ging während des Wachstums mit metabolischen Besonderheiten, die anhand verschiedener Merkmale des Blutplasmas festgestellt wurden, einher (KUHN u.a., 1998; FALKENBERG u.a., 1999). Bei mehreren Gewichtsklassen wurde für die DS ein signifikant niedrigerer Cholesteringehalt im Blut ermittelt. In der vorliegenden Arbeit sollte geprüft werden, ob bereits bei den neugeborenen Ferkeln der beiden Herkünfte Unterschiede im Wachstum, der Körperzusammensetzung und in speziellen Eigenschaften des Fettstoffwechsels vorhanden sind. 
2. Material und Methode

Die Ferkel wurden aus den 1. und 2. Würfen von 15 DL- und 19 DS-Sauen unterschiedlicher Abstammung bezogen. Unmittelbar nach der Geburt erfolgte eine Geschlechtsbestimmung und Erfassung des Geburtsgewichtes von allen lebend geborenen Ferkeln. Aus jedem Wurf wurden 2 männliche Ferkel, die dem durchschnittlichen Ferkelgewicht des Wurfes am besten entsprachen, für die Blutuntersuchungen ausgewählt. Durch Punktion der $V$, cava cranialis wurden von diesen Ferkeln Blutproben für die Bestimmung der Gehalte an Cholesterin und Triglyceriden gewonnen. Das Blut wurde für die Herstellung von Blutplasma zentrifugiert. Die Proben wurden bis zu den Untersuchungen bei $-20^{\circ} \mathrm{C}$ gelagert.

Die methodischen Beschreibungen für die Bestimmung der Plasmagehalte an Gesamtcholesterin, high density lipoproteins (HDL)-cholesterol, low and very low density lipoproteins (LDL+VLDL)-cholesterol sowie an Triglyceriden sind bei FALKENBERG u.a. (1999) gegeben.

Für die sich anschließende Körperanalyse wurde jeweils 1 Tier der beiden ausgewählten Ferkel verwendet. Nach der Narkotisierung und Tötung der Ferkel erfolgte eine manuelle Zerlegung der Tierkörper in Fleisch, Oberflächenfett, Innenfett, Knochen, Haut und innere Organe. Daraufhin wurden für die chemische Analyse der Tierkörperzusammensetzung vier Fraktionen gebildet; Fleisch und Oberflächenfett, verzehrbare Innereien und Innenfett, Knochen und Schwarten, Verdauungs- (leer)- und Geschlechtsorgane. Die chemische Analyse erfolgte hinsichtlich der Merkmale Wasser, Protein, Fett, Asche. Aus den Gehaltswerten der Einzelfraktionen wurde die chemische Zusammensetzung des Gesamtkörpers berechnet.

Die Wasser- bzw. Trockensubstanz- Bestimmung erfolgte durch Trocknung der Proben bei $105^{\circ} \mathrm{C}$. Die getrocknete Probe wurde für die Bestimmung des Fettgehaltes verwendet, wobei als Extraktionsmittel Petrolether zur Anwendung kam (ohne Säureaufschluß). Für die Bestimmung des Aschegehaltes wurden die Proben bei $600{ }^{\circ} \mathrm{C}$ im Muffelofen verascht. Als Gehaltswert für das Protein wurde der Trockensubstanzgehalt abzüglich der Gehalte von Fett und Asche zugrundegelegt (Differenz-Verfahren).

Für die Bestimmung des Fettzelldurchmessers wurden Proben aus dem subkutanen Fettgewebe in Höhe der 13./14. Rippe entnommen. Nach der Probenlagerung bei $70^{\circ} \mathrm{C}$ wurden die Messungen nach der bei WEGNER u.a. (1998) beschriebenen Methodik vorgenommen.

Die statistische Auswertung erfolgte varianzanalytisch mit der Prozedur GLM des Programm-Pakets SAS (Statistical Analysis System, 1985) unter Verwendung der Least-Square-Methode. Es wurde ein gemischtes Modell mit dem fixen Faktor der Rasse und dem zufälligen (Random) Faktor der Sau herangezogen. Als Signifikanzgrenze wurde ein Niveau von $\mathrm{P}<0,05$ zugrundegelegt.

\section{Ergebnisse}

Gegenüber den Landrasse-Tieren weisen die Sattelschweine in Verbindung mit einer geringeren Wurfgröße ein tendenziell höheres Geburtsgewicht auf (Tab. 1). Das Wägeergebnis der für die Schlachtkörperanalysen stichprobenartig ausgewählten Ferkel spiegelt sehr gut sowohl für das Geburts- als auch das Schlachtkörpergewicht die 
Tabelle 1

Körpergewicht und Schlachtkörperzusammensetzung bei neugeborenen Ferkeln (Body weight and carcass composition in newborn piglets)

\begin{tabular}{|c|c|c|c|c|c|}
\hline & & \multicolumn{2}{|c|}{$\begin{array}{l}D L \\
n=15\end{array}$} & \multicolumn{2}{|c|}{$\begin{array}{c}\text { DS } \\
n=19\end{array}$} \\
\hline & & LSM & SE & LSM & SE \\
\hline Geburtsgewicht') & $\mathrm{kg}$ & 1,36 & 0,02 & 1,42 & 0,02 \\
\hline Geburtsgewicht & g & 1363 & 59 & 1429 & 67 \\
\hline Schlachtkörpergewicht & g & 1025 & 42 & 1101 & 47 \\
\hline Fleisch & $\%$ & 44,00 & 0,66 & 44,23 & 0,58 \\
\hline Knochen & $\%$ & 33,57 & 0,53 & 33,41 & 0,47 \\
\hline Haut & $\%$ & 11,69 & 0,26 & 12,32 & 0,23 \\
\hline Oberflächenfett & $\%$ & 10,30 & 0,42 & 9,56 & 0,37 \\
\hline Innenfett & $\%$ & 0,70 & 0,04 & 0,66 & 0,03 \\
\hline Fettzelldurchmesser & $\mu \mathrm{m}$ & 12,97 & 0,64 & 12,94 & 0,55 \\
\hline
\end{tabular}

leichte Gewichtsdifferenz zugunsten der Sattelschweine wider. Zwischen den Ferkeln der beiden Rassen ließen sich keine bedeutenden Unterschiede in der Schlachtkörperzusammensetzung feststellen. Die Ferkel der Rasse DS neigen im Vergleich zu den Tieren der Rasse DL eher zu einem niedrigeren als zu einem höheren Fettanteil, Im Fettzelldurchmesser des subkutanen Fettgewebes bestehen bei den Tieren der beiden Rassen keine Unterschiede. Innerhalb der Schlachtkörpergewebe wurden Differenzen für den Hautanteil ermittelt, indem dieser bei den DS in der Tendenz höher ist $(\mathrm{P}=0,0749)$.

An Hand der chemischen Analyse ließen sich ebenfalls keine rassebedingten Unterschiede in den Gehalten an Wasser, Protein, Fett und Asche für die verschiedenen Körperfraktionen nachweisen. In Tabelle 2 sind die Gehaltswerte für den Gesamtkörper und den $m$. longissimus aufgefuhrt, die nur gering variieren.

Tabelle 2

Chemische Zusammensetzung des Gesamtkörpers und des $m$. longissimus bei neugeborenen Ferkeln (Chemical composition of the whole empty body and $m$. longissimus in newborn piglets)

\begin{tabular}{lrrrr}
\hline & \multicolumn{2}{c}{$\begin{array}{l}\text { DL } \\
\text { n=15 }\end{array}$} & \multicolumn{2}{c}{ DS } \\
& LSM & SE & LSM & SE \\
\hline Gesamtkörper & 79,66 & 0,38 & 80,18 & 0,34 \\
Wasser \% & 15,04 & 0,31 & 14,80 & 0,27 \\
Protein \% & 1,34 & 0,07 & 1,39 & 0,06 \\
Fett \% & 3,95 & 0,14 & 3,63 & 0,13 \\
Asche \% & & & & \\
m. longissimus & & & & \\
Wasser \% & 80,17 & 0,30 & 19,85 & 0,27 \\
Protein \% & 18,00 & 0,41 & 0,98 & 0,36 \\
Fett \% & 0,95 & 0,08 & 0,92 & 0,08 \\
Asche \% & 0,90 & 0,02 & 0,02 \\
\hline
\end{tabular}

Die Tabelle 3 enthält die Werte für den Anteil an inneren Organen, bezogen auf das Lebendgewicht (leer). Sowohl für die Stoffwechselorgane, wie Leber, Herz, Nieren, Lunge, als auch für die Intestinalorgane (Magen, Darm) wurden keine Unterschiede 
zwischen den Neugeborenen beider Rassen ermittelt.

Tabelle 3

Anteil an inneren Organen ${ }^{\text {") }}$ bei neugeborenen Ferkeln (Percentages of internal organs in newborn piglets)

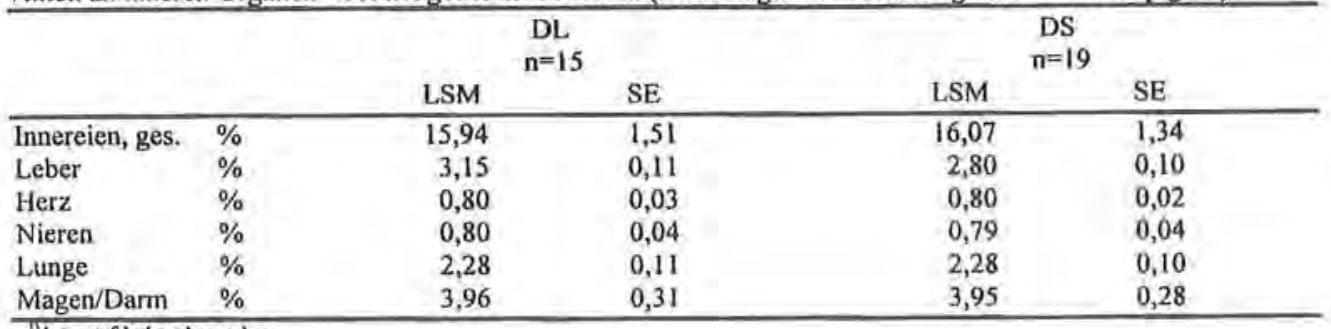

"bex. auf Lebendgew. leer

Der Blutplasmagehalt an Gesamt-Cholesterin ist bei den DS-Tieren gegenüber den DL-Tieren geringfügig höher (Tab. 4). Er steht mit dem signifikant höheren Gehalt an HDL-Cholesterin in Zusammenhang $(\mathrm{P}=0,0250)$. Der Gehalt an Triglyceriden ist nicht signifikant unterschiedlich bei den Neugeborenen beider Rassen.

Tabelle 4

Gehalte an Cholesterin und Triglyceriden im Blutplasma von neugeborenen Ferkein (Concentrations of cholesterol fractions and triglycerides in the blood plasma of newbom piglets)

\begin{tabular}{llllllll}
\hline & & \multicolumn{3}{c}{ DL } & \multicolumn{3}{c}{ DS } \\
& & $\mathbf{n}$ & LSM & SE & n & LSM & SE \\
\hline Cholesterin, ges. & $\mathrm{mg} / \mathrm{dl}$ & 29 & 52,89 & 4,22 & 32 & 57,15 & 4,02 \\
HDL-Chol. & $\mathrm{mg} / \mathrm{dl}$ & 20 & $9,93^{\circ}$ & 1,20 & 30 & $13,51^{\mathrm{b}}$ & 0,98 \\
VLDL+LDL-Chol. & $\mathrm{mg} / \mathrm{dl}$ & 20 & 43,41 & 5,05 & 29 & 44,74 & 4,19 \\
Triglyceride & $\mathrm{mg} / \mathrm{dl}$ & 29 & 45,55 & 6,76 & 32 & 57,84 & 6,43 \\
\hline
\end{tabular}

\section{Diskussion}

In den vorliegenden Untersuchungen konnten zwischen den Ferkeln der beiden Rassen DL und DS zum Zeitpunkt der Geburt weder Unterschiede im Körpergewicht noch in der Körperzusammensetzung ermittelt werden. Die ausgewachsenen Tiere der DS weisen im Vergleich zu der auf einen hohen Muskelfleischanteil gezüchteten Rasse einen höheren Fettanteil im Schlachtkörper auf. In früher durchgeführten Untersuchungen wurde für diese Tiere im Vergleich zu Tieren der Rasse DL während der Mast ein langsameres Wachstum und zum Zeitpunkt der Schlachtung ein um ca. $13 \%$ (abs.) höherer Gesamtfettgehalt festgestellt (KUHN u.a., 1998). Von Sauen obeser und primitiver Rassen, wie Ossabaw und Chinesische Meishan, ist bekannt, dass im Vergleich zu fettärmeren Herkünften bereits die Föten besonders ab dem letzten Trächtigkeitsabschnitt ein niedrigeres Körpergewicht und eine veränderte Körperzusammensetzung aufweisen (KASSER u.a., 1981; HOFFMANN u.a., 1983; STONE u.a., 1985; HACKMAN u.a., 1996). Die gleiche Feststellung wurde für Ferkel von Sauen getroffen, die auf eine hohe Rulckenspeckdicke selektiert worden waren (MERSMANN u.a., 1984; STONE u.a., 1985). In der Regel wurde bei Nachkommen von fetten Sauen ein höherer Trockensubstanzgehalt bei erhöhtem Fettgehalt festgestellt.

Von HAUSMANN u.a. (1983) wurde weiterhin nachgewiesen, dass der höhere Fettansatz der Föten und Ferkel von fetten Sauen mit Besonderheiten in der Adipozytengröße verbunden war. So wurden im subkutanen Fettgewebe von Föten der Rasse 
Chinesische Meishan größere Adipozyten gefunden als bei Föten einer fleischreichen Herkunft. HERPIN u.a. (1993) fanden bei der Rasse Chinesische Meishan im Vergleich zu weniger fettreichen Tieren eine größere Anzahl von Adipozyten mit hohem Durchmesser.

LÖHRKE u.a. (2000) zeigten an 30 Tage alten männlichen Ferkeln der Rassen Pietrain (PI) und DS, dass der biologische Mechanismus, der zu Variationen in der Fettgewebeentwicklung führt, über Veränderungen in der ektopischen Expression von TNF $\alpha$ (Tumornekrose-Faktor $\alpha$ ) und PPAR $\gamma$ (Peroxisomenproliferatoraktivierter Rezeptor $\gamma$ ) verläuft und eine Selektion nach verringerter Fettbildung auf die Regulation der Aktivität der Gene TNF $\alpha$ und PPAR $\gamma$ zielt. Ein deutlich höherer intrazellulärer TNF $\alpha$ Spiegel steht in Übereinstimmung mit der geringeren Fettgewebeentwicklung bei den PI-Tieren, weil TNF $\alpha$ lipolytisch wirkt und das Zelltodprogramm in reifen Adipozyten anregen kann (REID u.a., 1991).

In einigen Untersuchungen wurden bei neugeborenen Ferkeln von fetteren Sauen auch höhere Proteingehalte in der Muskulatur festgestellt (HOFFMANN u.a., 1983; STONE u.a., 1985). Damit wird deutlich, dass das genetische Potential für einen höheren Fettansatz in Relation zum Proteinansatz erst postnatal zum Ausdruck kommen kann. Da sich in den vorliegenden Untersuchungen bei den Neugeborenen noch keine Unterschiede in der Körperzusammensetzung nachweisen ließen, ist anzunehmen, dass den Tieren der beiden Rassen Stoffwechselmechanismen zugrundeliegen, die Differenzen im Fett- und Proteinansatz erst im postnatalen Wachstumsabschnitt entstehen lassen. Es ist bekannt, dass bestimmte genetische Effekte in betonter Weise erst postnatal phänotypisch umgesetzt werden (ROBISON, 1972; BERESKIN u.a., 1974).

In der Literatur wird für Föten und Ferkel von obesen im Vergleich zu fleischreichen Sauen auch von rassespezifischen metabolischen sowie endokrinen Differenzen berichtet (MARTIN u.a., 1985; HERPIN u.a., 1993). Die vorliegenden Untersuchungen weisen für die Neugeborenen der DS-Schweine einen signifikant höheren HDL-Cholesterin-Gehalt im Blutplasma aus. Dieser höhere Gehaltswert steht in Übereinstimmung mit den während des postnatalen Wachstums der DS gefundenen signifikant niedrigeren LDL- und VLDL-Werten (FALKENBERG u.a., 1999). Hohe CholesterinWerte bei den Neugeborenen könnten sich vorteilhaft auf die Überlebensrate auswirken. Aus verschiedenen Untersuchungen ist bekannt, dass das Cholesterin-Niveau während der neonatalen Phase in positiver Weise mit dem Wachstum und der Entwicklung des Nervensystems korreliert (SCHOKNECHT u.a.., 1994; POND und MERSMANN, 1995). Das HDL-Cholesterin wirkt auch beim Schwein atherosklerotischen Veränderungen der Blutgefäße entgegen und übt bereits in frühen Altersabschnitten schützende Funktionen aus (HACKMAN u.a., 1995).

Möglicherweise sind die Deutschen Sattelschweine für die Untersuchung der beim Menschen bekannten Atherosklerose ein sehr gut geeignetes Tiermodell.

Literatur

BERESKIN, B.; HETZER, H.O.; PETERS, W.H.; NORTON, H.W.

Genetic and maternal effects on pre-weaning traits in crosses of high- and low-fat lines of swine, $\mathrm{J}$, Anim, Sci. 39 (1974), 1-10

FALKENBERG, H.; KUHN, G.; HARTUNG, M.; LANGHAMMER, W.; WOLF, C.: Verlauf von biochemischen Kennwerten im Blut von Schweinen mit unterschiedlicher Fettansatzleistung. Arch. Tierz., Dummerstorf 42 (1999), 149-159 
HACKMAN, A.M.; POND, W.G.; MERSMAN, H.J.; WONG, W.W.; KROOK, L.P.; ZHANG, S.:

Obese pigs fed a high cholesterol diet from birth to 2 months are less susceptible than lean pigs to atherosclerosis. J. Nutrit. 126 (1996), 564-573

HAUSMAN, G.J.; CAMPION, D.R.; THOMAS, J.B.;

Adipose tissue cellularity and histochemistry in fetal swine as affected by genetic selection for high or low backfat. J, Lipid Res. 24 (1983), 223

HERPIN, P.; DIVIDICH, J.L.; AMARAL, N.:

Effect of selection for lean tissue growth on body composition and physiological state of the pig at birth. J. Anim. Sci. 71 (1993), 2645-2653

HOFFMAN, E.C; WANGSNESS, P.J.; HAGEN, D.R.; ETHERTON, T.D.:

Fetuses of lean and obese swine in late gestation: body composition, plasma hormones and muscle development. J. Anim. Sci. 57 (1983), 609-619

KASSER, T.R.; MARTIN, R.J.; CAHAGAN, J.H.; WANGSNESS, P.J.:

Fasting plasma hormones and metabolites in feral and domestic newborn pigs. J. Anim. Sci. 53 (1981) (Suppl. 2), 420

KUHN, G.; HARTUNG, M.; FALKENBERG, H.; NÜRNBERG, G.; LANGHAMMER, M.; SCHWERIN, M.; ENDER, K.:

Wachstum, Körperzusammensetzung und Fleischbeschaffenheit von im Fettansatz genetisch differenten Schweinen. Arch. Tierz., Dummerstorf 40 (1997), 345-355

KUHN, G.; NÜRNBERG, K.; FIEDLER, I.; FALKENBERG, H; NÜRNBERG, G.; ENDER, K.;

Körperzusammensetzung und Muskelstruktur von genetisch differenten Schweinen in Abhängigkeil vom MHS.Status, Arch. Tierz., Dummerstorf 41 (1998), 589-596

LÖHRKE, B.; LANGHAMMER, M.; DIETL, D.:

Expression of the Transcriptions Factors, Peroxisome Proliferator-Activated Receptor and CCAAT Enhancer Binding Protein $\alpha$, and of Tumor Necrosis Factor $\alpha$ at the Protein Level in Subcutaneous Tissues from Pietrain and Saddleback Piglets: Implications for the Regulation of Adipogenesis. J. Anim. Sci. 2000 (eingereicht)

MARTIN, R.J.; RAMSAY, T,G; CAMPION, D.R; HAUSMAN, G.J.: Fetal hormone and metabolite levels in lean and obese pigs. Growth 49 (1985), 400-407

MERSMANN, H.J.; POND, W.G.; STONE, R.T., YEN, J.T.; LINDVALL, R.N.:

Factors affecting growth and survival of neonatal genetically obese and lean swine: cross fostering experiments, Growth 48 (1984), 209

POND, W.G.; MERSMANN, H.J.:

Genetically diverse pig models in nutrition research related to lipoprotein and cholesterol metabolism. In: M. TUMBELSON and L. SCHOCK (Ed.) International symposia of swine in biomedical research. pp 843-864. Plenum Press, New York. 1995

REID, T.; RAMESHA, C.S.; RINGOLD, G.M.:

Resistance to killing by tumor necrosis factor in an adipocyte cell line caused by a defect in arachidonic acid biosynthesis. J. Biol. Chem. 266 (1991), 16580-16586

ROBISON, O.W.: Maternal effects in swine. J. Anim. Sci. 35 (1972), 1303-1315

SCHOKNECHT, P.A.; EBNER, S.; POND, W.G.; ZHANG, S.; McWHINNEY, V.; WONG, W.W.; KLEIN, P.D.; DUDLEY, M.; GODDARD-FINEGOLD, J.; MERSMANN, H.J.:

Dietary cholesterol supplementation improves growth and behavioral response of pigs selected for genetically high and low serum cholesterol. J. Nutrit. 124 (1994), 305-314

STONE, R.T.; CAMPION, D.R.; KLINDT, J.; MARTIN, R.J.:

Blood parameters and body composition in fetuses from reciprocal crosses of genetically lean and obese swine. Proc. of the society for experimental biology and medicine, 180 (1985), 191-195

WEGNER, J.; ALBRECHT, E; ENDER, K:

Morphologische Aspekte des subkutanen und intramuskulären Fettzellwachstums. Arch. Tierz., Dummerstorf 41 (1998), 313-320

Anschrift der Verfesser

Dr. GERDA KUHN, Dr. HEINZ FALKENBERG, Dr. MARTINA LANGHAMMER, Dr. MARGITTA HARTUNG, Dr. JOCHEN WEGNER, Prof. Dr, habil. KLAUS ENDER

Forschungsinstitut fulr die Biologie landwirtschaftlicher Nutztiere (FBN)

Wilhelm-Stahl-Allee 2

D-18196 Dummerstorf 